\title{
Prevalência e epidemiologia da leishmaniose visceral em cães e humanos, na cidade de Cuiabá, Mato Grosso, Brasil
}

\author{
Prevalence and epidemiology of visceral leishmaniasis in dogs and humans in the city Cuiaba, \\ Mato Grosso, Brazil
}

\author{
Arleana do Bom Parto Ferreira de Almeida ${ }^{I}$ Adriane Jorge Mendonça ${ }^{I I}$ \\ Valéria Régia Franco Sousa ${ }^{I I}$
}

\section{RESUMO}

A leishmaniose visceral é uma doença infecciosa endêmica em diversas regiões do Brasil, incluindo o Estado de Mato Grosso. Em Cuiabá, no período de janeiro de 2006 a dezembro de 2008, foi realizada investigação dos casos caninos de infecção por Leishmania sp. por meio de sorologia e citologia, enfocando a sua distribuição geográfica $e$ correlacionando a ocorrência de casos de leishmaniose visceral humana. De 150 cães com suspeita da infecção, 57 (38\%) foram sorológicos ou parasitologicamente positivos. Observouse soroprevalência significativa $(P \leq 0,05)$ nos bairros com renda per capita baixa, além de maior número de casos caninos na regional leste; no entanto, observou-se uma distribuição difusa da doença canina na cidade de Cuiabá, enquanto os casos humanos originaram principalmente na regional norte do município. Esse fato sugere que há risco em todo o município, devendo ser realizados novos estudos soroepidemiológicos, assim como a distribuição vetorial, que promovam maior conhecimento da infecção canina por Leishmania sp, de forma a se gerar medidas adequadas para o controle da doença.

Palavras-chave: Leishmania (Leishmania) infantum chagasi, georreferenciamento, cão, Mato Grosso, Brasil.

\section{ABSTRACT}

Visceral leishmaniasis is an infectious disease endemic in several regions of Brazil, including the state of Mato Grosso. In Cuiabá, from January 2006 to December 2008, a research was performed in canine cases of infection with Leishmania sp. by serology and cytology, focusing on its geographical distribution, correlating to the occurrence of human visceral leishmaniasis cases. From 150 dogs with suspected infection, 57 (38\%) were serological or parasitologically positive. Seroprevalence was observed $(P \leq 0.05)$ in districts with low per capita income, besides a larger number of canine cases in the East regional, but there was a diffuse distribution of the canine disease in the city of Cuiabá, in contrast to cases focused mainly on human in the northern municipality. This suggests risk throughout the municipality and further epidemiological studies should be carried out, as well as vector distribution, to promote better understanding of canine infection by Leishmania, in order to promote appropriate measures for disease control.

Key words: Leishmania (Leishmania) infantum chagasi, georeferencing, dog, Mato Grosso, Brazil.

\section{INTRODUÇÃO}

A leishmaniose visceral é uma doença infecciosa crônica frequentemente letal, causada nas Américas pela Leishmania (Leishmania) infantum chagasi e transmitida pelo díptero Lutzomya longipalpis e Lutzomya cruzi (MISSAWA \& LIMA, 2006). Os primeiros registros de casos autóctones no Estado de Mato Grosso ocorreram no ano de 1973 (BARUFFA \& CURY, 1973). Com a transformação ambiental e ocupação desordenada dos grandes centros urbanos, no ano de 1998, registrou-se o primeiro caso de leishmaniose visceral humana na região metropolitana de Cuiabá (MESTRE \& FONTES, 2007). O cão é o principal reservatório doméstico de $\boldsymbol{L}$.

IPrograma de Pós-graduação em Pesquisa Clínica em Doenças Infecciosas, Instituto de Pesquisa Evandro Chagas da Fundação Oswaldo Cruz, Manguinhos, RJ, Brasil.

${ }^{I}$ Departamento de Clínica Médica Veterinária, Universidade Federal de Mato Grosso (UFMT). Av. Fernando Correa da Costa, S/No, Bairro Boa Esperança, 78060-900, Cuiabá, MG. E-mail: regia@ufmt.br. Autor para correspondência. 
(L.) i. chagasi, sendo o responsável pela manutenção desse agente na área urbana. Os cães infectados, especialmente os assintomáticos, são potencial fonte de infecção para o vetor, representando, em áreas endêmicas para a doença, a maior fonte de transmissão do parasito para o homem (ASHFORD, 1996).

Em muitas regiões brasileiras, a leishmaniose visceral (LV) canina e humana ocorre endemicamente (FEITOSA et al., 2000). Em Mato Grosso, os relatos de LV canina e da presença da $\mathbf{L}$. longipalpis são recentes (MISSAWA \& LIMA, 2006) e em Cuiabá os inquéritos determinaram prevalência variável de 3,4 a $64 \%$ (MOURA et al., 1999; ALMEIDA et al., 2009). O estudo da distribuição geográfica tem sido ferramenta utilizada em estudos epidemiológicos relacionados à $L V$, permitindo identificar padrões espaciais de morbidade e/ou mortalidade e fatores sócio-econômicos e ambientais associados, bem como descrever a difusão das doenças, gerando informações sobre os agravos e objetivando sua predição, sua prevenção e seu controle (DANTAS-TORRES \& BRANDÃO-FILHO, 2006).

Diante desse quadro e da crescente notificação de casos de LV humana, foi realizada, no período de janeiro de 2006 a dezembro de 2008, uma investigação dos cães com anticorpos anti-Leishmania e/ou com formas amastigotas em aspirado de medula óssea e linfonodo, enfocando a distribuição geográfica no Município de Cuiabá, área de cerrado matogrossense, no Brasil, e contrapondo os dados com os casos de LV humana ocorridos na cidade.

\section{MATERIAL E MÉTODOS}

No período de janeiro de 2006 a dezembro de 2008, no Setor de Clínica Médica de Pequenos Animais do Hospital Veterinário da Universidade Federal de Mato Grosso, foram atendidos 150 cães com suspeita clínica de LV, além de contactantes de cães diagnosticados com a infecção, sendo classificados clinicamente em sintomáticos, oligossintomáticos, apresentando de um a três sinais clínicos, e assintomáticos (MANCIANTI et al., 1998). Um questionário foi aplicado aos proprietários, visando a obter dados referentes aos aspectos epidemiológicos da doença canina.

Amostras sanguíneas obtidas por meio de punção jugular, aspiradas de medula óssea do esterno e de linfonodo poplíteo, foram coletadas para realização de imunofluorescência indireta e análise citológica, respectivamente, após consentimento dos responsáveis. A imunofluorescência indireta foi desenvolvida de acordo com as recomendações do fabricante (Biomanguinhos ${ }^{\circledR}$, FIOCRUZ), considerando o título igual ou maior a 1:40 reagente. A análise citológica dos aspirados se deu após utilizar-se a coloração rápida do tipo Romanowsky em microscopia óptica (100x).

Os casos caninos foram registrados e notificados as autoridades sanitárias (Centro de Controle de Zoonoses e Secretaria Estadual de Saúde). Esses casos, adicionados aos casos humanos de 2002 a 2008 (SES-MT, 2009), foram georreferenciados por meio do Sistema de Posicionamento Global (GPS Gramin $\left.^{\circledR}\right)$ e distribuídos geograficamente com auxílio do programa Google Earth 5.0. e Adobe Photoshop CS3 ${ }^{\circledR}$.

Os dados de soroprevalência foram comparados ao sexo, à faixa etária, ao quadro clínico e à localização geográfica dos cães examinados, por meio do teste de qui-quadrado e teste exato de Fisher, pelo programa Epi info 3.3.2 (CDC, EUA), sendo considerados estatisticamente significativos $\mathrm{P} \leq 0,05$.

\section{RESULTADOS E DISCUSSÃO}

Nesta pesquisa dos 150 cães com suspeita clínica de LV, 56 (37,3\%) foram sororreagentes. Em aspirados de medula óssea e linfonodo dos animais sororreagentes, foram diagnosticadas formas amastigotas de Leishmania sp. em 32 (21,3\%) e 31 $(20,7 \%)$ cães, respectivamente. Em um cão soronegativo, foram encontradas formas parasitárias no aspirado de linfonodo, totalizando 57 cães infectados e prevalência de $38 \%$.

Estudos anteriores demonstraram variável prevalência da doença canina na cidade de Cuiabá, com 64\% dos cães sororreagentes em 1999 (MOURA et al., 1999) e 8,4\% no período de 1998 a 2005 (MESTRE \& FONTES, 2007), sendo utilizados imunoensaio enzimático (ELISA) e imunofluorescência indireta (IFI). $\mathrm{O}$ mais recente trabalho realizado no município menciona prevalência de $3,4 \%$ pela técnica de IFI (ALMEIDA et al., 2009). Essa variação poderia estar relacionada ao ponto de corte adotado no teste sorológico ou à forma de seleção dos cães e da definição de população adotada (JULIÃO et al., 2007). Neste estudo, o ponto de corte não justifica a diferença de prevalências, já que se utilizou o adotado pelos outros autores $(1: 40)$, mas o fator determinante foi a população estudada, direcionada a cães com suspeita da doença, decorrendo em viés de seleção.

No presente estudo, três apresentaram título de 1:40, oito $1: 80$, quatorze $1: 160$, quinze $1: 320$ e dezesseis 1:640. A possível ocorrência de reações cruzadas foi descartada com a observação de formas amastigotas de Leishmania sp. nos aspirados de medula óssea e linfonodo, o que ressalta a importância 
da associação de métodos diagnósticos mais específicos, como o parasitológico, na detecção do agente etiológico. Estudos relatam não haver predisposição etária, sexual ou racial quanto à infecção por Leishmania sp. em cães (GONTIJO \& MELO, 2004). Apesar da maior ocorrência de fêmeas infectadas, não houve diferença estatisticamente significativa (Tabela 1). A faixa etária mais frequentemente relatada foi entre um e três anos de idade, concordando com outros autores que afirmam ser mais descrita em cães adultos devido ao longo período de incubação do parasito, que, em média, é de dois meses, além do maior tempo de exposição ao vetor (GÁLLEGO, 2004). Entretanto, FRANÇA-SILVA et al. (2003) observaram que os cães, independente da idade, têm a mesma probabilidade de contrair leishmaniose, não excluindo a importância das outras faixas etárias na epidemiologia da doença canina

Quanto à distribuição racial observada neste estudo, os cães infectados em sua maioria eram sem raça definida (18), seguida da raça boxer (10), porém sem diferença estatisticamente significativa, fato que não predispõe esses dois grupos raciais à infecção, apesar da descrição de maior susceptibilidade da raça boxer (FRANÇA-SILVA et al., 2003). Outras raças sororreagentes foram Pit Bull (8), Pinscher (6), Teckel (6), Rottweiler (2), Pastor Alemão (4), Poodle (1), Pointer (1) e Bull Terrier (1). Apesar de todos os cães serem susceptíveis à infecção por Leishmania, tem se associado a maior frequência de casos aos cães de porte grande, pois estes têm a finalidade de guarda, habitando ambiente peridomiciliar e ficando assim mais expostos ao vetor (FEITOSA et al., 2000).

Os cães infectados encontravam-se sintomáticos e oligossintomáticos (Tabela 1), fato justificado por se tratar de ambiente de assistência veterinária. As alterações clínicas mais observadas foram as dermatológicas $(96,5 \%)$, dermatite furfurácea, alopecia e úlceras cutâneas, seguidas por linfadenomegalia $(70,2 \%)$, alterações oftálmicas $(64,9 \%)$, como conjuntivite, onicogrifose $(63,1 \%)$, emagrecimento progressivo $(57,9 \%)$, apatia $(49,1 \%)$, esplenomegalia $(38,6 \%)$, atrofia muscular $(35,1 \%)$ e hepatomegalia $(21 \%)$, condizentes com estudos anteriores (ALMEIDA et al., 2005).

Com os dados obtidos em cada consulta e questionário, foi possível identificar que 50 cães eram provenientes da cidade de Cuiabá e sete de cidades vizinhas à capital, Várzea Grande (2), Santo Antônio do Leverger (1) e Nossa Senhora do Livramento (1), municípios da Baixada Cuiabana e endêmicos para LV. $\mathrm{O}$ encontro de cães infectados pertencentes a municípios circunvizinhos destaca a importância do trânsito livre de animais na disseminação do agente, bem como do rastreamento dos casos caninos atendidos em clínicas e hospitais veterinários no controle e na prevenção da doença (JULIÃO et al., 2007). Na distribuição espacial dos cães infectados e domiciliados na cidade de Cuiabá, verificou-se que 33 eram provenientes da regional leste, nove da norte, seis da oeste e dois da sul (Figura 1), sendo observada diferença estatisticamente significativa $(\mathrm{P} \leq 0,05)$ entre a regional leste e a sul. A distribuição dos casos por bairros de acordo com a renda per capita (PMC, 2004) encontra-se inserida na tabela 2.

$\mathrm{Na}$ análise da distribuição de cães infectados de acordo com o abairramento por renda per capita, se observou diferença estatística entre os bairros com a renda baixa e os demais. Contudo, esse resultado deve ser analisado com cautela, já que um pequeno número de cães era proveniente desses locais, onde o acesso à

Tabela 1 - Relação entre sexo, idade e sinais clínicos dos cães com leishmaniose visceral no período de janeiro de 2006 a dezembro de 2008.

\begin{tabular}{|c|c|c|c|c|c|}
\hline & Variável & № Cães & Positivos (\%) & $X^{2}$ & $\mathrm{P}$ \\
\hline \multirow{2}{*}{ Sexo } & Macho & 77 & $24(31,2)$ & 2,6 & 0,10 \\
\hline & Fêmea & 73 & $33(45,2)$ & & \\
\hline \multirow{4}{*}{ Idade } & $=1$ ano & 11 & $3(27,3)$ & 4,1 & 0,39 \\
\hline & $>1-3$ anos & 28 & $20(71,4)$ & & \\
\hline & $>3-6 a n o s$ & 40 & $16(40)$ & & \\
\hline & $=6$ anos & 53 & $16(30)$ & & \\
\hline \multirow{4}{*}{ Sinais clínicos } & Desconhecida & 7 & $2(28.6)$ & & \\
\hline & Sintomático & 94 & $47(50)$ & 8,6 & $0,003 *$ \\
\hline & Oligossintomático & 45 & $10(22,2)$ & & \\
\hline & Assintomático & 11 & $0(0,0)$ & & \\
\hline
\end{tabular}

\footnotetext{
* significativo em nível de $5 \%$ de probabilidade estatística pelo teste $\chi^{2}$.
} 


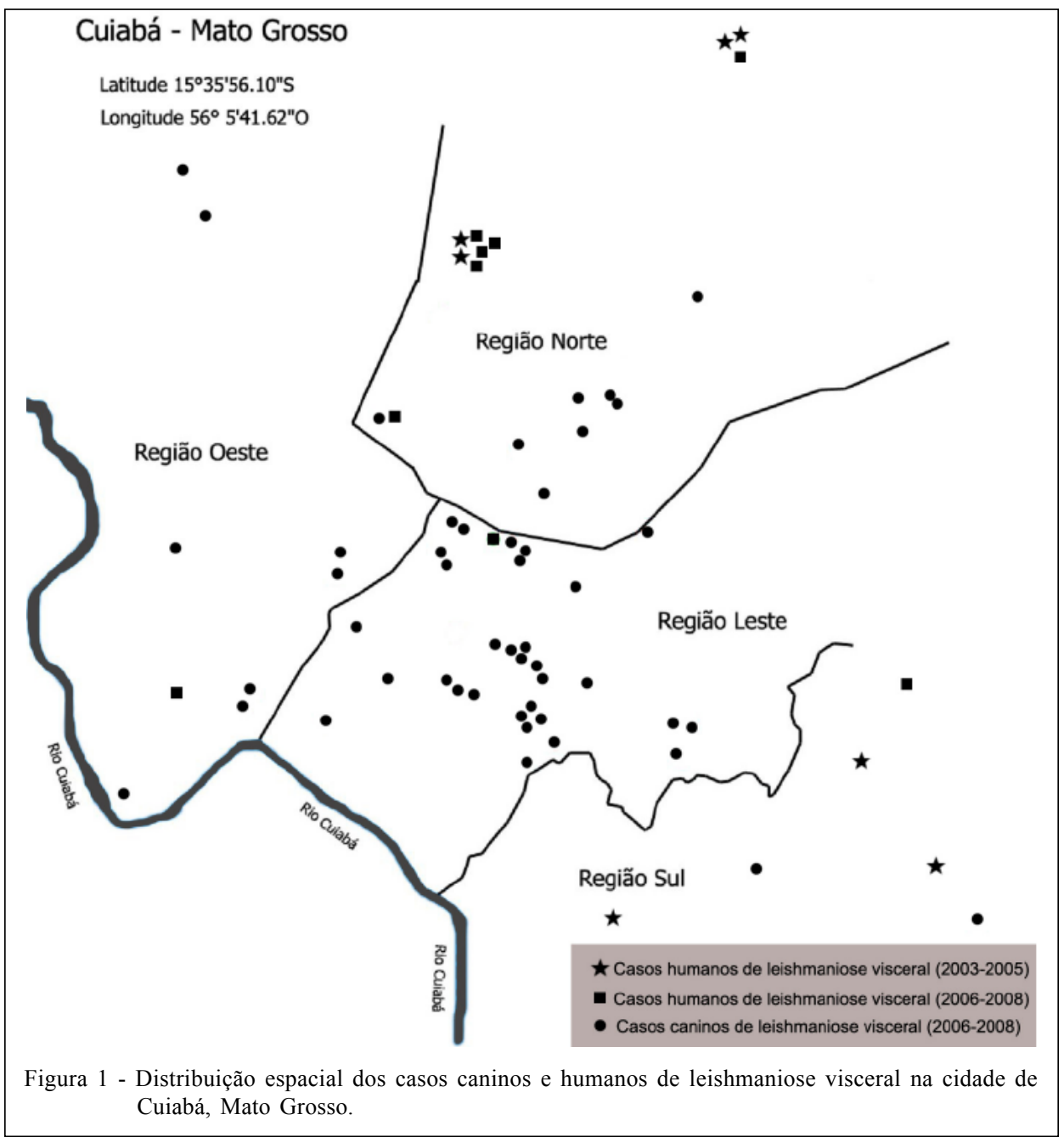

informação sobre as leishmanioses, assim como a procura por assistência veterinária, parece ser mais restrito. No entanto, observa-se um elevado número de cães infectados residentes em bairros de classe média a alta, advertindo para mudança no perfil da doença nos últimos anos.

$\mathrm{Na}$ análise espacial dos casos caninos pesquisados, observou-se uma distribuição difusa da doença no município, com maior número descrito na regional leste (Figura 1), devido à proximidade com o hospital. Entretanto, pôde-se observar que a doença canina encontra-se distribuída por toda a capital, contrapondo a distribuição de casos humanos nos últimos sete anos, registrados na sua maioria na área rural e periferia da regional norte, área com precária infraestrutura higiênico-sanitária decorrente da ocupação de áreas por população de baixa renda e criação de loteamentos, provocando a destruição de ecótopos silvestres, que contribuem para a instalação da LV (GONTIJO \& MELO, 2004). No presente trabalho, a avaliação da distribuição geográfica da doença demonstra crescente ocorrência dos casos humanos em área urbana do município, além de infecção canina. Entretanto, por se tratar de estudo realizado com base em casos caninos atendidos na rotina veterinária e não de inquérito, pode não corresponder à verdadeira situação da infecção, mas alerta para distribuição difusa de LV canina, enquanto que a doença no homem ainda está concentrada na regional norte.

De acordo com os dados obtidos durante a consulta clínica, 49 cães viviam em ambiente urbano, e apenas 14 tinham acesso esporádico à zona rural. Esses dados confirmam o processo de urbanização da doença no município, semelhantemente às observações de ALMEIDA et al. (2009). Vinte cães com a infecção tinham suas residências contíguas à mata remanescente, $24 \mathrm{a}$ terrenos baldios, 14 a rios, córregos ou represas e em 13 dos casos não existia vegetação próxima, dados

Ciência Rural, v.40, n.7, jul, 2010. 
Tabela 2 - Frequência de casos de leishmaniose canina nos bairros de Cuiabá classificados de acordo com a renda per capita, no período de janeiro de 2006 a dezembro de 2008.

\begin{tabular}{lccc}
\hline Faixa de renda $^{*}$ & Cães positivos & Cães negativos & Total \\
\hline Baixa $^{\mathrm{A}}$ & $04(2,9 \%)$ & $00(0,0 \%)$ & $04(2,9 \%)^{\mathrm{a}^{* *}}$ \\
Média-Baixa $^{\mathrm{B}}$ & $15(10,9 \%)$ & $29(21 \%)$ & $44(31,9 \%)^{\mathrm{b}}$ \\
Média $^{\mathrm{C}}$ & $07(5,1 \%)$ & $25(18,1 \%)$ & $32(23,2 \%)^{\mathrm{b}}$ \\
Média-Alta $^{\mathrm{b}}$ & $19(13,8 \%)$ & $25(18,1 \%)$ & $44(31,9 \%)^{\mathrm{b}}$ \\
Alta $^{\mathrm{E}}$ & $05(3,6 \%)$ & $09(6,5 \%)$ & $14(19,1 \%)^{\mathrm{b}}$ \\
\hline
\end{tabular}

*Faixa de Renda em salário mínimo: A: <2,91; B: de 2,91 a 5,65; C: de 5,66 a 11,65; D: de 11,66 a 21,94; E: >21,94.

${ }^{* *}$ Dados seguidos de letras diferente na vertical indicam $\mathrm{P}=0,05$ pelo teste exato de Fisher.

estes não estatisticamente significativos $(\mathrm{P} \leq 0,05)$. A importância de áreas com vegetação remanescente na periferia das cidades é aspecto relatado em várias pesquisas como determinante para ocorrência da infecção, em virtude de ambiente rico em matéria orgânica propício para manutenção do vetor (MISSAWA \& LIMA, 2006). Além disso, análise multivariada tem demonstrado que a situação sócioeconômica precária, o aumento da vegetação e a infecção canina prévia estão associados à ocorrência de casos humanos (COSTA, 2008).

Cerca de $87,7 \%$ dos cães infectados eram nascidos no Município de Cuiabá, área de cerrado, bioma que, segundo MISSAWA \& LIMA (2006), se encontra $\boldsymbol{L}$. longipalpis e $\mathbf{L}$. cruzi. De acordo com LEMOS et al. (2004), essas espécies, em especial a $\boldsymbol{L}$. longipalpis, apresenta ampla distribuição, sendo encontrada em espaços geográfico, climático e social variados, nas regiões norte, nordeste, centro-oeste e sudeste, em geral em regiões de vegetação rasteira ou arbustiva, comum em áreas de cerrado.

\section{CONCLUSÃO}

A distribuição difusa da LV canina em Cuiabá indica maior risco da doença e demonstra a importância de inquéritos epidemiológicos mais abrangentes no município, tendo em vista o incremento de casos humanos na capital, além da adoção de medidas eficazes de prevenção.

\section{COMUNICAÇÃO PESSOAL}

SECRETARIA ESTADUAL DE SAÚDE DO ESTADO DE MATO GROSSO, 2009 (SES-MT, 2009). Centro Político Administrativo, Palácio Paiaguás, Bloco D, CEP: 78049-902, Cuiabá, Mato Grosso. Telefone: (65) 3613-5000.

\section{COMITÊ DE ÉTICA E BIOSSEGURANÇA}

A presente pesquisa foi aprovada pelo Comitê de Ética em Pesquisa Animal (CEPA) - UFMT, documento n. 242
23108.043642/08-3, sendo realizada de acordo com as normas éticas.

\section{AGRADECIMENTOS}

Ao Conselho Nacional de Desenvolvimento Científico e Tecnológico (CNPq)/DECIT - MS e à Fundação de Apoio à Pesquisa do Estado de Mato Grosso (FAPEMAT), pelo auxílio financeiro.

\section{REFERÊNCIAS}

ALMEIDA, M.A.O. et al. Clinical and serological aspects of visceral leishmaniasis in Northeast Brazilian dogs naturally infected with Leishmania chagasi. Veterinary Parasitolology, v.127, p.227-232, 2005. Disponível em: <http:// w w w. $\mathrm{s}$ c i e $\mathrm{n}$ c e d i r e c t . c o m / science?_ob=ArticleURL\&_udi=B6TD7-4F 1 HCGB$1 \&$ _user $=686451 \&$ _coverDate $=02 \% 2$ F $28 \% 2$ F $2005 \&$ alid $=1335368103 \&$ rdoc $=1 \&$ fmt $=$ high\&_orig $=$ search \&_cdi $=5$ $191 \&$ _sort $=$ r\&_docanchor $=\&$ view $=$ c\&_ct $=27 \&$ acct $=\bar{C} 0000$ $37559 \&$ \& version $=1 \&$ \&_urlversion $=0 \&$ \& userid $=68$ $6451 \& \mathrm{md} \overline{5}=7 \mathrm{f} 982 \mathrm{~d} 309 \mathrm{c} 1 \mathrm{~d} 1 \mathrm{dda} 35 \mathrm{c} 2 \mathrm{~b} 3 \mathrm{cdcba} 0 \mathrm{fe} 19>$. Acesso em: 10 jun. 2007. doi:10.1016/j.vetpar.2004.10.010.

ALMEIDA, A.B.P.F. et al. Inquérito soroepidemiológico de leishmaniose canina em áreas endêmicas de Cuiabá, Estado de Mato Grosso. Revista da Sociedade Brasileira de Medicina Tropical, v.42, n.2, p.156-159, 2009. Disponível em: http:// www.scielo.br/scielo.php?script $=$ sci arttext\&pid $=$ S003786822009000200012. Acesso em: 15 set. 2009. doi: 10.1590/ S0037-86822009000200012.

ASHFORD, R.W. Leishmaniasis reservoirs and their significance in control. Clinics in Dermatology, v.14, p.523532, 1996. Disponível em: <http://www.sciencedirect.com/ science? ob=ArticleURL \& udi=B6T5G-3W3NF8XF\&_user $=686451 \&$ _coverDate=10\%2F $31 \% 2$ F 1996 \&_alid $=1335379093 \&$ rdoc $=1 \&$ fmt $=$ high\&_orig $=$ search \&_cdi $=$ $5002 \&$ s ort $=$ r\&_docañ chor $=\&$ vi e w $=$ c \& _ ct $=$ $609 \&$ acct $=$ C $000037559 \&$ _version $=1 \&$ _urlVersio $\mathrm{n}=0$ \&_userid $=686451 \& \mathrm{md} 5=\mathrm{d} 0351 \mathrm{~d} 3 \mathrm{cf} 8264 \mathrm{bfb} 9237 \mathrm{f} 6091899 \mathrm{f} 3 \mathrm{~d} 2>$. Acesso em: maio, 2008. doi:10.1016/0738-081X(96)00041-7.

BARUFFA, G.; CURY, P. Contribuição ao estudo do calazar em Mato Grosso. Revista Patologia Tropical, v.2, p.345-361, 1973.

Ciência Rural, v.40, n.7, jul, 2010. 
COSTA, C.H.N. Characterization and speculations on the urbanization of visceral leishmaniasis in Brazil. Caderno de Saúde Pública, v.24, n.12, p.2959-2963, 2008 Disponível em: <http://www.scielo.br/scielo.php?pid=S0102311X2008001200027\&script $=$ sci_arttext $>$. Acesso em: 09 fev. 2008. doi: 10.1590/S0102-311X2008001200027.

DANTAS-TORRES, F.; BRANDÃO-FILHO, S.P. Expansão geográfica da leishmaniose visceral no Estado de Pernambuco. Revista da Sociedade Brasileira de Medicina Tropical, v.39, n.4, p.352-356, 2006. Disponível em: <http:// www.scielo.br/scielo.php?script $=$ sci arttext\&pid $=\mathrm{S} 0037$ $86822006000400007>$. Acesso em: 13 out. 2008. doi: $10.1590 / \mathrm{S} 0037-86822006000400007$

FEITOSA, M.M. et al. Aspectos clínicos de cães com leishmaniose visceral no município de Araçatuba, São Paulo (Brasil). Clinica Veterinária, v.28, p.36-44, 2000.

FRANÇA-SILVA, J.C. et al. Epidemiology of canine visceral leishmaniosis in the endemic area of Montes Claros Municipality, Minas Gerais State, Brazil. Veterinary Parasitology, v.111, p.161-173, 2003. Disponível em: <http:/ / w w w. s c i e n c e d i r e c t. c o m / science?_ob=ArticleURL\&_udi=B6TD7-476K 7CD$4 \&$ user $=\overline{6} 86451 \&$ coverDate $=02 \% 2 \mathrm{~F} 13 \% 2 \mathrm{~F} 2003 \&$ alid $=$ $1335392080 \&$ rdoc $=1 \&$ fmt $=$ high \&_orig $=$ search \&_cdi $=51$ $91 \&$ sort $=r \&$ docanchor $=\& v i e w=c \&$ ct $=17 \&$ acct $=C$ $000037559 \&_{\text {__version }}=1 \&$ \&_urlVersion $=0 \&_{-}$_useri $\mathrm{d}=686451 \& \mathrm{md} \overline{5}=7 \mathrm{fa} 0 \mathrm{ed} 03831 \overline{\mathrm{bcc}} 209424039 \mathrm{~d} 82 \mathrm{ec} 048<$ Acesso em: 13 out. 2008. doi:10.1016/S0304-4017(02)00351-5.

GÁLLEGO, M. Zoonosis emergentes por patógenos parasitos: las leishmaniosis. Review Scientific and Technical Office International des Epizooties, v.23, n.2, p.661-676, 2004. Disponível em: <http://www.oie.int/boutique/extrait/ 661676gallegoesp.pdf>. Acesso em: 14 jul. 2008.

GONTIJO, C.M.F.; MELO, M.N. Leishmaniose visceral no Brasil: quadro atual, desafios e perspectivas. Revista Brasileira de Epidemiologia, v.7, n.3, p.338-349, 2004. Disponível em: $<$ http://www.scielo.br/s cielo.php?pid=S 1415 $790 X 2004000300011 \&$ script $=$ sci arttext $>$. Acesso em 26 jul. 2008. doi: 10.1590/S1415-790X 2004000300011 .

JULIÃO, F.S. et al. Investigação de áreas de risco como metodologia complementar ao controle da leishmaniose visceral canina. Pesquisa Veterinária Brasileira, v.27, p.319-324, 2007. Disponível em: $<$ http://www.scielo.br/scielo.php?script=sci arttext\&pid=S0100736X2007000800001>. Acesso em: 13 jul. 2008. doi: 10.1590/ S0100-736X2007000800001.
LEMOS, J.C. et al. Encontro de Lutzomyia longipalpis na área de implantação da usina hidrelétrica Capim Branco I, na bacia do rio Araguari, no município de Uberlândia, Minas Gerais - Brasil. Revista Caminhos de Geografia, v.12, p.186-198, 2004. Disponível em: <http://www.caminhosdegeografia.ig.ufu.br/ viewarticle.php?id=96>. Acesso em: 17 jan. 2009.

MANCIANTI, F. et al. Studies on canine leishmaniasis control. I. Evolution of infection of different clinical forms of canine leishmaniasis following antimonial treatment. Transactions of the Royal Society of Tropical Medicine and Hygiene, v.82, p.566-567, 1998. Disponível em: <http://www.sciencedirect.com/ science?_ob=ArticleURL\&_udi=B 75GP-4C0KBHR$1 \mathrm{~J} \mathrm{~B} \mathrm{\&} \mathrm{u} \mathrm{s} \mathrm{e} \mathrm{r}=686451 \&$ c o v e r D a t e $=$ $08 \% 2$ F $31 \% 2$ F $1988 \&$ \& a 1 id $=1335404118 \&$ rdoc $=1 \&$ \&mt $=$ high \&_orig=search\&_cdi=13100\& docanchor $=\&$ view-c\&_ct $=67 \&$ \& a c t $=$ C 000 $037559 \&$ version $=1 \&$ urlversion $=0 \&$ userid $=68$ $6451 \& \mathrm{md} 5=\mathrm{da} 8192 \mathrm{efebb} 960 \mathrm{~d} 9 \mathrm{e} 39 \mathrm{c} 367 \mathrm{~b} 3 \mathrm{c} 24 \mathrm{daa} 7>$. Acesso em: 23 maio, 2008. doi:10.1016/0035-9203(88)90510-X.

MESTRE, G.L.C.; FONTES, C.J.F. A expansão da epidemia de leishmaniose visceral no Estado de Mato Grosso, 1998-2005. Revista da Sociedade Brasileira de Medicina Tropical, v.40, n.1, p.42-48, 2007. Disponível em: <http://www.scielo.br/ s c i e lo.php ? s c ript $=$ s ci arttext \& pid $=$ S 0037 $86822007000100008 \& \operatorname{lng}=\mathrm{en}>$. Acesso em: 29 ago. 2008. doi: $10.1590 / \mathrm{S} 0037-86822007000100008$.

MISSAWA, N.A.; LIMA, G.B.M. Distribuição espacial de Lutzomyia longipalpis (Lutz \& Neiva, 1912) e Lutzomyia cruzi (Mangabeira, 1938) no Estado de Mato Grosso. Revista da Sociedade Brasileira de Medicina Tropical, v.39, n.4, p.337-340, 2006. Disponível em: <http://www.scielo.br/ s c i e lo.php ? s cript $=$ sci_art text \& pid $=$ S 0037 86822006000400004>. Acesso em: 29 ago. 2008. doi: $10.1590 / \mathrm{S} 0037-86822006000400004$.

MOURA, S.T. et al. Diagnóstico de leishmaniose canina na área urbana do município de Cuiabá, Estado de Mato Grosso, Brasil. Brazilian Journal Veterinary Research and Animal Science, v.36, n.2, p.101-102, 1999. Disponível em: <http:// www.scielo.br/scielo.php?script $=$ sci_arttext\&pid $=\mathrm{S} 1413$ 95961999000200009>. Acesso em: 13 abr. 2008. doi: 10.1590/ S1413-95961999000200009.

PREFEITURA MUNicipal DE CUiAbÁ. Perfil socioeconômico de Cuiabá, volume II. Cuiabá: Instituto de Pesquisa e Desenvolvimento Urbano, 2004. 407p. 